\title{
A Virtual Model of Spring Reverberation
}

\author{
Stefan Bilbao and Julian Parker
}

\begin{abstract}
The digital emulation of analog audio effects and synthesis components, through the simulation of lumped circuit components has seen a large amount of activity in recent years; electromechanical effects have seen rather less, primarily because they employ distributed mechanical components, which are not easily dealt with in a rigorous manner using typical audio processing constructs such as delay lines and digital filters. Spring reverberation is an example of such a system-a spring exhibits complex, highly dispersive behavior, including coupling between different types of wave propagation (longitudinal and transverse). Standard numerical techniques, such as finite difference schemes are a good match to such a problem, but require specialized design and analysis techniques in the context of audio processing. A model of helical spring vibration is introduced, along with a family of finite difference schemes suitable for time domain simulation. Various topics are covered, including numerical stability conditions, tuning of the scheme to the response of the model system, numerical boundary conditions and connection to an excitation and readout, implementation details, as well as computational requirements. Simulation results are presented, and full energy-based stability analysis appears in an Appendix.
\end{abstract}

Index Terms-Artificial reverberation, finite difference schemes, musical acoustics, physical modeling, springs, virtual analog.

\section{INTRODUCTION}

$\mathbf{T}$ HE emulation of analog electronic components used in sound synthesis and processing, or "virtual analog," as it has come to be known, has seen a large amount of interest in recent years-see, e.g., [1]-[4]. The case of electromechanical effects has been somewhat less investigated at the level of physical models, though there has been some work on plate reverberation [5]-[7], the Leslie speaker [8], and the application to be discussed here, spring reverberation [9], [10]. Not surprisingly, because of the presence of distributed components, modeling techniques are more in line with those applied to acoustic musical instruments than in the case of analog circuit components, which are conveniently modeled as lumped.

Spring reverberation [11]-[13] was originally intended as a cost-effective means of approximating room reverberation, through the dense set of echoes created in the repeated re-

Manuscript received March 29, 2009; revised August 17, 2009. Current version published April 14, 2010. This work was supported by the Engineering and Physical Sciences Research Council, U.K., under Grant C007328/1, and the Academy of Finland under Project 122815. The associate editor coordinating the review of this manuscript and approving it for publication was Dr. Udo Zoelzer.

S. Bilbao is with the Acoustics and Fluid Dynamics Group/Music subject area, University of Edinburgh, Edinburgh EH8 9AD, UK (e-mail: sbilbao@staffmail.ed.ac.uk).

J. Parker is with the Department of Signal Processing and Acoustics, Helsinki University of Technology, FI-02015 TKK Finland (e-mail: julian.parker@tkk.fi).

Digital Object Identifier 10.1109/TASL.2009.2031506 flections of an input signal, injected at one end, from the terminations. Though, due to the very nature of wave propagation on a spring, the results can never sound like those of a real room, the effect has its own appeal and audience, and various digital emulation systems have appeared. Some are based on impulse responses drawn from physical units, and others on delay line structures [14]. The former approach suffers from a lack of flexibility (i.e., one is bound to an existing database of recorded impulse responses), and the second from the lack of a strong physical justification for the use of delay line structures as a model of the dynamics of highly dispersive structures (such as springs). Mainstream simulation techniques, such as finite difference schemes, which directly simulate an underlying system of differential equations, address both of these difficulties. Computational costs are higher for such methods, but recent increases in computing power on commercially available hardware justify an exploration of such methods.

In Section II, a simple model of helical spring vibration is presented, accompanied by a discussion of dispersive effects, as well as the means by which the spring may be driven, and output read. Section III continues with the development of an implicit finite difference scheme, and various practical implementation details are discussed. Numerical results appear in Section IV. A full analysis of numerical stability appears in the Appendix. Some of the material in this article is expanded from a brief treatment of the topic in [15].

\section{HELICAL SPRING VibRATION}

There are many models of the vibration of a helical structure; the best known is the family which originates with the work of Wittrick [16], written as a system in 12 variables. For springs used in a reverberation setting, the Wittrick model faithfully models phenomena occuring at frequencies far outside the range of human auditory perception (i.e., in the $\mathrm{MHz}$ range), and is certainly overkill in audio applications. A much simpler model is the following:

$$
\begin{aligned}
u_{t t}= & -\frac{E r^{2}}{4 \rho}\left(u_{x x x x}+2 \epsilon^{2} u_{x x}+\epsilon^{4} u\right) \\
& +\frac{E \epsilon}{\rho}\left(\zeta_{x}-\epsilon u\right)-2 \sigma_{t} u_{t} \\
\zeta_{t t}= & \frac{E}{\rho}\left(\zeta_{x x}-\epsilon u_{x}\right)-2 \sigma_{l} \zeta_{t} .
\end{aligned}
$$

Here, $u(x, t)$ is the transverse displacement of the spring in a single polarization away from a rest position, as a function of arc length $x \in[0, L]$ along the spring, for some total unwound spring length $L$, and time $t \geq 0$, and $\zeta(x, t)$ is displacement in the longitudinal direction. Subscripts $x$ and $t$ indicate differentiation with respect to the spatial and temporal variables, respectively. See Fig. 1. $\rho$ is material density, $E$ is Young's modulus, $r$ 


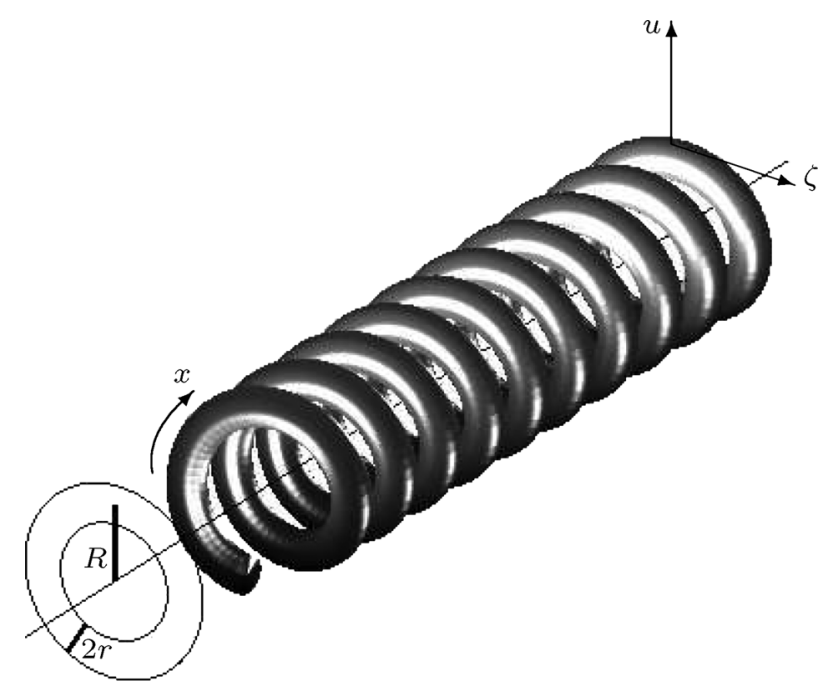

Fig. 1. Helical spring, of coil radius $R$, and wire radius $r$. Displacements $\zeta$, in the longitudinal direction, and $u$, in a transverse direction are as indicated; both depend on $x$, arc length as measured along the spring axis.

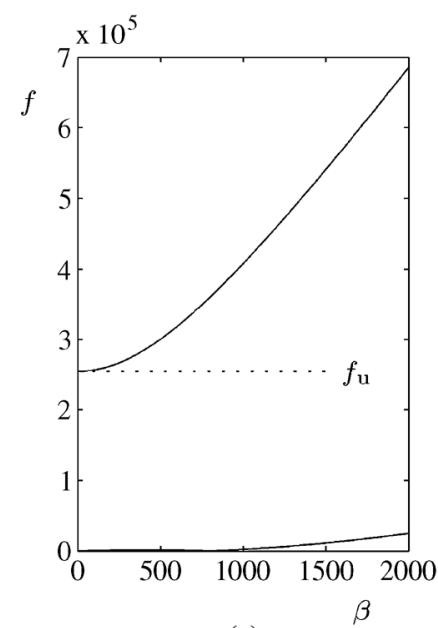

(a)

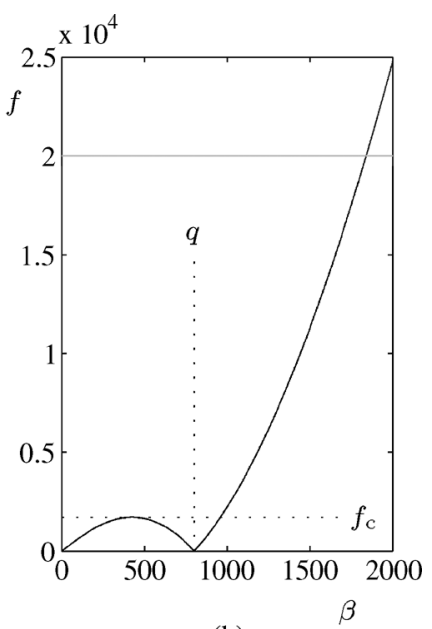

(b)
Fig. 2. (a) Dispersion relations $f(\beta)$ for system (2), with $\kappa=0.05, \gamma=2000$ and $q=800$; the minimum of the upper curve occurs at approximately $f_{\mathrm{u}}$, far above the range of human hearing. (b) A close-up view of the lower dispersion relation, with features related to the parameters $q$ and $f_{c}$. The upper threshold of human hearing, at $f=20 \mathrm{kHz}$ is indicated by a solid gray line.

is the radius of the wire (assumed of circular cross section), and the parameter $\epsilon \approx 1 / R$ is a measure of the curvature of the spring, where $R$ is the coil radius. The terms involving the parameters $\sigma_{t}$ and $\sigma_{l}$ model loss, and in their absence, system (1) is that presented in [17]. These terms are chosen as a simple "ad hoc" model of loss, but a physically reasonable one in the sense that they lead to strict dissipation (see the Appendix). When $\epsilon=0$, and under lossless conditions, system (1) reduces to the equation of motion of an ideal bar (in $u$ ), and the 1-D wave equation (in $\zeta$ ). The assumptions leading to system (1) are that the wire is thin, under minimal tension, and that the helix angle of the spring is small; in addition, motion in only one transverse polarization is modeled-for springs of moderate curvature, the two polarizations are nearly degenerate, so it suffices to examine one of the two.

\section{A. Scaled Form}

If the non-dimensional variables $x^{\prime}=x / L, u^{\prime}=\epsilon u$, and $\zeta^{\prime}=\zeta / L$ are introduced, system (1) may be written, after removing primes, as

$$
\begin{aligned}
u_{t t}= & -\kappa^{2}\left(u_{x x x x}+2 q^{2} u_{x x}+q^{4} u\right) \\
& +q^{2} \gamma^{2}\left(\zeta_{x}-u\right)-2 \sigma_{t} u_{t} \\
\zeta_{t t}= & \gamma^{2}\left(\zeta_{x x}-u_{x}\right)-2 \sigma_{l} \zeta_{t}
\end{aligned}
$$

over the unit interval $x \in[0,1]$, and where

$$
\kappa=\sqrt{\frac{E}{\rho}} \frac{r}{2 L^{2}} \quad \gamma=\frac{1}{L} \sqrt{\frac{E}{\rho}} \quad q=\epsilon L .
$$

Crudely speaking, $\kappa$ is a measure of the stiffness of the system, $\gamma$ of the longitudinal wave velocity, and $q$ the curvature-all have important perceptual correlates, as will be seen shortly.

\section{B. Dispersion Relations}

As system (2) is linear and shift-invariant, in the lossless case (i.e., when $\sigma_{t}=\sigma_{l}=0$ ), its characteristics may be summarized by a dispersion relation, written in terms of an angular frequency $\omega$ and a wave number $\beta$. This may be obtained by Fourier analysis of (2), or by inserting traveling wave components $u(x, t)=U e^{j(\omega t+\beta x)}$ and $\zeta(x, t)=Z e^{j(\omega t+\beta x)}$, of amplitudes $U$ and $Z$, to yield

$$
\left[\begin{array}{cc}
\omega^{2}-\kappa^{2}\left(\beta^{2}-q^{2}\right)^{2}-q^{2} \gamma^{2} & j q^{2} \gamma^{2} \beta \\
-j \gamma^{2} \beta & \omega^{2}-\gamma^{2} \beta^{2}
\end{array}\right]\left[\begin{array}{l}
U \\
Z
\end{array}\right]=\left[\begin{array}{l}
0 \\
0
\end{array}\right]
$$

Nontrivial solutions occur when the determinant of the above matrix is zero, leading to the characteristic equation

$$
\left(\omega^{2}-\gamma^{2} \beta^{2}\right)\left(\omega^{2}-\kappa^{2}\left(\beta^{2}-q^{2}\right)^{2}-\gamma^{2} q^{2}\right)-\gamma^{4} q^{2} \beta^{2}=0 .
$$

This dispersion relation possesses four solutions $\omega(\beta)$, grouped as two pairs. The two relations of positive sign are plotted in Fig. 2, as functions $f(\beta)$, where $f=\omega / 2 \pi$ is frequency in Hertz, for typical choices of system parameters found in reverberation devices. These curves are of a very different form from those found in non-twisted structures such as straight bars and strings, in that the lower relation is not monotonic - as such, it is possible for components of distinct wavelengths to have the same temporal frequency. The lower curve is the one of primary interest in reverberation. It possesses a zero at wave number $\beta=q$, corresponding to rigid body rotation of the spring, and 
reaches a maximum at $f_{\mathrm{c}}$ at approximately $\beta=q / 2$. By inserting this value of $\beta$ in the characteristic (4), and solving for $\omega$, this transition frequency $f_{\mathrm{c}}$ may be written as

$$
f_{\mathrm{c}} \approx \frac{3 \kappa q^{2}}{8 \pi \sqrt{5}}
$$

and is a useful design parameter if one is trying to calibrate a physical model to a measured spring response (i.e., if one does not have access to the physical parameters directly), as it is easily visible in a spectrogram. See Fig. 2(b).

The upper curve gives rise to frequencies which lie outside the range of human auditory perception, for springs of dimensions of interest in audio applications; indeed, the minimum of the upper curve occurs at a frequency $f_{\mathrm{u}}$ which is approximately given by

$$
f_{\mathrm{u}} \approx \frac{\gamma q}{2 \pi}=\frac{\sqrt{E / \rho}}{2 \pi R} \gg 20000 \mathrm{~Hz}
$$

see Fig. 2(a). As such, it would be desirable to avoid modeling such solutions altogether. Unfortunately, however, in the context of strict physical modeling, it is difficult to uncouple such a solution from system (2) without destroying the form of the primary curve, which is, of course, of crucial importance in giving a spring reverb unit its characteristic sound. In a framework based on signal processing constructions such as delay lines or digital waveguides [9], one could attempt to make use of the dispersion curve directly, as a means of designing terminating digital filters. One should keep in mind, however, that these filter designs will necessarily be of high order, and that the underlying dynamics are somewhat obscured by the assumption of dispersionless wave propagation (i.e., in delay lines). In fact, wave propagation in springs is highly dispersive-indeed, even if the spring is essentially straight, with $q=0$, system (2) reduces to the equation of motion of an ideal bar, in $u$. Such concerns are the rationale behind the more direct simulation approach to modeling carried out in the remainder of this paper.

\section{Input/Output and Spring Boundary Conditions}

Boundary conditions have not yet been specified for system (2); first one must have a model of the termination, as input and output are related to the motion of magnetic beads connected to the spring at its endpoints-in some units, the beads are connected by thin wires to a rigid, and perhaps lossy support structure. Though a full model of these elements would be rather extensive, in many units their motion will be aligned with the longitudinal motion of the spring (i.e., $\zeta$ ) at the spring endpoints. See Fig. 3.

A model of the motion of the beads is as follows:

$$
\begin{aligned}
\ddot{w}_{\mathrm{L}}+2 \sigma_{\mathrm{L}} \dot{w}_{\mathrm{L}}+\omega_{\mathrm{L}}^{2}\left(w_{\mathrm{L}}-\frac{1}{2} \zeta(0, t)\right) & =F(t) \\
\ddot{w}_{\mathrm{R}}+2 \sigma_{\mathrm{R}} \dot{w}_{\mathrm{R}}+\omega_{\mathrm{R}}^{2}\left(w_{\mathrm{R}}-\frac{1}{2} \zeta(1, t)\right) & =0 .
\end{aligned}
$$

Here, $w_{\mathrm{L}}(t)$ and $w_{\mathrm{R}}(t)$ are the displacements (non-dimensional) of the beads at the driving and pick-up ends of the

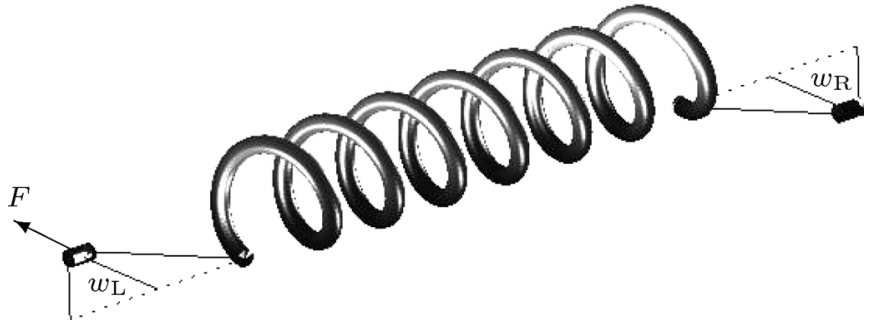

Fig. 3. Connections between a spring to mass-spring systems, constrained to move in the longitudinal direction, with position $w_{\mathrm{L}}$ (at the driving end) and $w_{\mathrm{R}}$ at the pickup. The mass at the driving end is driven by a force-like excitation $F$, aligned with longitudinal displacement at the spring endpoint.

spring, respectively, and dots indicate total time derivatives; $\omega_{\mathrm{L}}$ and $\omega_{R}$ are the associated natural frequencies of oscillation of the beads, and $\sigma_{\mathrm{L}}$ and $\sigma_{\mathrm{R}}$ damping parameters. Not indicated above are the mass ratios $M_{\mathrm{L}}$ and $M_{\mathrm{R}}$ of the beads relative to the total spring mass. In addition, $F=F(t)$, of dimensions $1 / \mathrm{s}^{2}$, represents an excitation (the audio input, perhaps after undergoing some prefiltering).

The system (2) requires the specification of three boundary conditions at each of $x=0$ and $x=1$; these may be related to the terminating mass-spring systems, through dynamic considerations, or energy analysis as follows:

$$
\begin{aligned}
& x=0,1: u=0 \quad \text { or } \quad u_{x x x}+q^{2} u_{x}=0 \\
& x=0,1: u_{x}=0 \quad \text { or } \quad u_{x x}+q^{2} u=0 \\
& x=0: \zeta=0 \quad \text { or } \quad u-\zeta_{x}-\frac{M_{\mathrm{L}} \omega_{\mathrm{L}}^{2}}{2 \gamma^{2}}\left(w_{\mathrm{L}}-\zeta\right)=-\frac{M_{\mathrm{L}}}{\gamma^{2}} F \\
& x=1: \zeta=0 \quad \text { or } \quad u-\zeta_{x}+\frac{M_{\mathrm{R}} \omega_{\mathrm{R}}^{2}}{2 \gamma^{2}}\left(w_{\mathrm{R}}-\zeta\right)=0 .
\end{aligned}
$$

Combinations of the first two pairs of conditions, which refer only to the transverse displacement, may be viewed as extensions of the normal clamped, simply supported or free conditions of an ideal bar [18]. In this paper, it is assumed, for simplicity, that the first of each pair holds, though there is no reason that the other conditions could not be used. The second of each pair in the latter conditions relates the spring displacement to those of the beads-notice that the mass ratios $M_{\mathrm{L}}$ and $M_{\mathrm{R}}$ intervene.

Under any of the above conditions, the system as a whole is strictly dissipative, under transient conditions; when driven, the state may be bounded in terms of the energy supplied by the source. See the Appendix.

\section{TIME-Domain MethodS}

A standard approach in simulation, and one which is especially well-suited to problems defined in over a single spatial coordinate, or in 1-D, such as the spring system presented here, is to make use of a finite difference approximation. Because of the presence of two distinct sets of natural frequencies in different ranges (see Section II-B); however, one must take great care to avoid problems associated with numerical dispersion, which can be extremely severe in such cases; explicit methods [10] can only give reasonable results at extremely high sample rates. For this reason, an implicit parameterized family of methods 
will be presented here; the interest of such schemes is in obtaining a good approximation to the primary dispersion relation of the model system at an audio sample rate. The conventional notion of numerical accuracy is not so useful in audio applications, as it refers to the behavior of a scheme in the limit of low frequencies - a scheme with a high formal order of accuracy may perform very poorly in the upper range of the spectrum. The idea is to develop a scheme which approximates the solution well over the entire spectrum, and not just in the limit of low frequencies, even though its formal accuracy may be low (here, second order); such methods are in line with so-called spectral-like schemes, which have been explored in mainstream problems [19].

\section{A. Difference Operators}

A grid function $f_{l}^{n}$ is intended as an approximation to a continuous function $f(x, t)$ at spatial locations $x=l h$ and $t=n k$, where $h$ is the spacing between adjacent grid points, and where $k$ is a time step (and $f_{s}=1 / k$ is the sample rate, normally set $a$ priori in audio applications as a typical rate such as $f_{s}=$ $44.1 \mathrm{kHz}$, etc.). For simplicity, the grid spacing is assumed to divide the unit interval evenly, such that $h=1 / N$, for some integer $N$.

In order to represent a scheme for (2) in a compact form, it is useful to introduce the following operator notation: for a given grid function $f_{l}^{n}$, the basic forward and backward shift operators in time and space are defined as

$$
\begin{aligned}
e_{t+} f_{l}^{n} & =f_{l}^{n+1} \\
e_{t-} f_{l}^{n} & =f_{l}^{n-1} \\
e_{x+} f_{l}^{n} & =f_{l+1}^{n} \\
e_{x-} f_{l}^{n} & =f_{l-1}^{n} .
\end{aligned}
$$

Basic difference operators may be defined in terms of these shifts as

$$
\begin{aligned}
\delta_{t t} & =\frac{1}{k^{2}}\left(e_{t+}-2+e_{t-}\right) \\
\delta_{t} . & =\frac{1}{2 k}\left(e_{t+}-e_{t-}\right) \\
\mu_{t, \alpha} & =\alpha+\frac{1-\alpha}{2}\left(e_{t+}+e_{t-}\right) \\
\delta_{x+} & =\frac{1}{h}\left(e_{x+}-1\right) \\
\delta_{x-} & =\frac{1}{h}\left(1-e_{x-}\right) \\
\delta_{x x} & =\delta_{x+} \delta_{x-} \\
\delta_{x x x x} & =\delta_{x x} \delta_{x x} .
\end{aligned}
$$

The difference operators $\delta_{t t}$ and $\delta_{t}$. are approximations to a second time derivative, and a first time derivative, respectively. $\mu_{t, \alpha}$ is an approximation to the identity operator, parameterized by the real number $\alpha$-when $\alpha=1$, it is a simple identity operation, and when $\alpha=0$, it is an average of two values neighboring the point of application of the operator-averaging operators such as this allow for the tuning of implicit methods [15]. The operators $\delta_{x+}$ and $\delta_{x-}$ are approximations to a first spatial derivative, and $\delta_{x x}$ and $\delta_{x x x x}$ to second and fourth spatial derivatives, respectively. All except $\delta_{x+}$ and $\delta_{x-}$ are secondorder accurate.

\section{B. Implicit Family of Schemes}

Consider the following approximation to system (2), written in a compact operator form

$$
\begin{aligned}
\left(1+\eta \kappa k \delta_{x x}\right) \delta_{t t} u= & -\kappa^{2}\left(\delta_{x x x x}+2 \bar{q}^{2} \delta_{x x}+\bar{q}^{4}\right) u \\
& +q^{2} \gamma^{2} \mu_{t, \alpha}\left(\delta_{x-} \zeta-u\right)-2 \sigma_{t} \delta_{t} . u \\
\left(1+\theta \kappa k \delta_{x x}\right) \delta_{t t} \zeta= & \gamma^{2} \mu_{t, \alpha}\left(\delta_{x x} \zeta-\delta_{x+} u\right)-2 \sigma_{l} \delta_{t} . \zeta .
\end{aligned}
$$

Here, $u=u_{l}^{n}$ and $\zeta=\zeta_{l}^{n}$ are grid functions which serve as approximations to $u(x, t)$ and $\zeta(x, t)$. This is an implicit family of schemes, depending on four free parameters, $\alpha, \bar{q}, \eta$, and $\theta$. In particular, the parameter $\bar{q}$ must be chosen as an approximation to $q$. This family of schemes contains, as special cases, many "obvious" choices of finite difference scheme-when $\alpha=1$ and $\eta=\theta=0$, e.g., it reduces to an explicit scheme. The terms involving the free parameters are the simplest choices available, provided that 1) the scheme is to remain a two-step method, and 2) the update, at a given point, depends on values at nearest neighbors only, simplifying the analysis of boundary conditions.

This scheme is formally second-order accurate-notice that the terms of coefficient $\eta$ and $\theta$ are scaled by $k$, and thus vanish in the limit of a high sample rate-furthermore, the operator $\mu_{t, \alpha}$ approximates the identity for any value of $\alpha$, and $\bar{q}$ is a second-order accurate approximation to $q$. Thus, at high frequencies, all solutions to the above scheme, for any choice of the parameters, will converge to the same result, provided the scheme is stable. In the numerical context, such formal accuracy refers to the behavior of the scheme in the limit of low frequencies, but such a point of view is not particularly useful in an audio context, where one would ideally obtain a good approximation to the model system over the entire range of audio frequencies; the free parameters are chosen such that the scheme as a whole remains formally consistent with the underlying model system, but allow for such a "tuning"—-see Section III-D.

\section{Stability Conditions}

A full stability analysis is postponed until the Appendix; though frequency domain (von Neumann) analysis [20] is possible, energy methods [21], [22] allow for a much more compact analysis, as well as the extraction of suitable termination conditions.

Necessary stability conditions for the scheme above are as follows. When $0 \leq \alpha \leq 1 / 2$, and when $\bar{q}$ is chosen as

$$
\bar{q}=\frac{2}{h} \sin (q h / 2)
$$



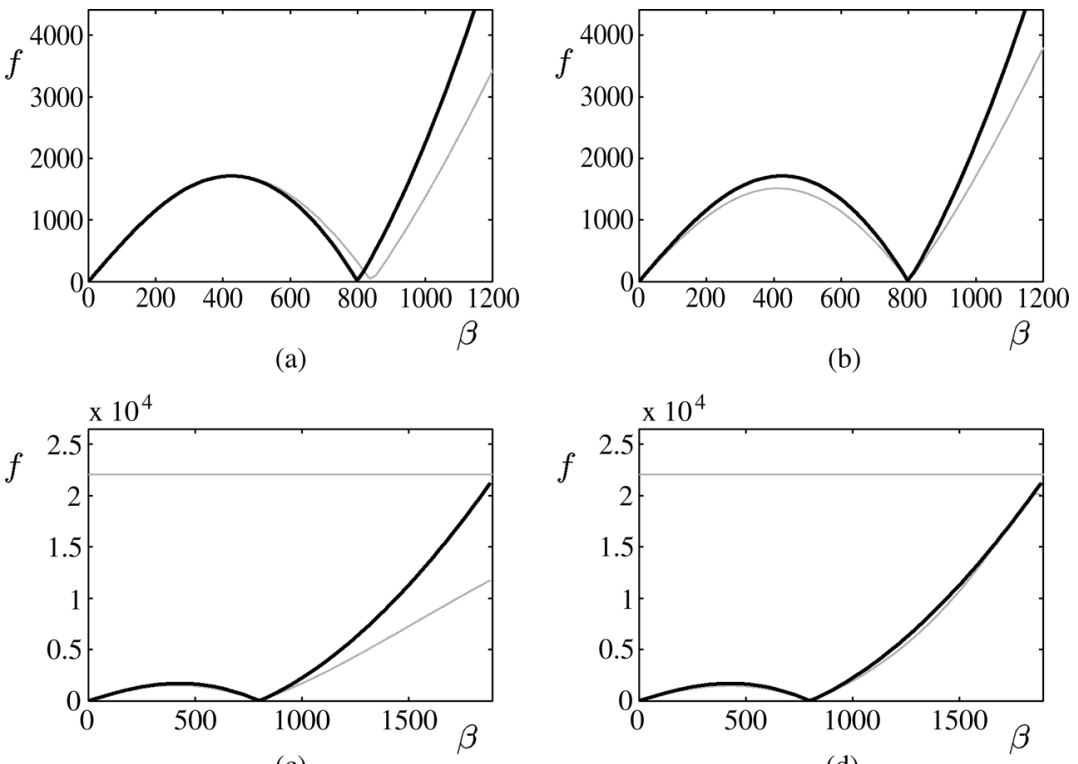

(c)

(d)

Fig. 4. Numerical dispersion relation for scheme (10) (as a gray line) compared with that of the model system (2) (as a black line), of physical parameters as given in the caption to Fig. 2, under various choices of the scheme coefficients. (a) In the default configuration, with $\bar{q}=q$, and $\eta=\theta=0$, (b) with $\bar{q}=2 \sin (q h / 2) / h$ and $\eta=\theta=0$, showing the alignment of the zero of the numerical dispersion relation with that of the model system, and (c), the same configuration as in (b), compared with (d), where $\bar{q}=2 \sin (q h / 2) / h, \eta=0.47$ and $\theta=0.000343$, and the numerical dispersion exhibits low error at high frequencies.

the grid spacing $h$ must satisfy the following conditions in terms of $k, \eta$, and $\theta$, and the model parameters $\kappa, q$ and $\gamma$

$$
\begin{aligned}
& h \geq 2 \gamma k \sqrt{\theta^{+}} \\
& h \geq \sqrt{\kappa k\left(2 \eta^{+}+\sqrt{4\left(\eta^{+}\right)^{2}+(1+|\cos (q h)|)^{2}}\right)}
\end{aligned}
$$

where here, $\eta^{+}=(\eta+|\eta|) / 2$, and $\theta^{+}=(\theta+|\theta|) / 2$. In fact, it is a good idea to choose the grid spacing as close to the above bounds as possible. Such bounds imply a maximum number of grid points to be used to discretize the unit interval; using fewer grid points, for a given sample rate results in a reduction in computational cost, but at the expense of potentially severe numerical dispersion effects - this is characteristic of all numerical methods for time-dependent or dynamical systems [15].

\section{Numerical Dispersion and Tuning}

In order to see the effect of the various parameters on the behavior of scheme (10), it is useful to examine plots of numerical dispersion relations, as shown in Fig. 4. (Such numerical dispersion relations may be obtained from scheme (10) in a manner similar to the continuous case, by inserting a solution of the form $u_{l}^{n}=e^{j(\beta l h+\omega n k)}$, corresponding to a discrete wave-like component of the solution to (10).)

Beginning from the simplest default configuration, in which $\alpha=1 / 2, \bar{q}=q$, and $\eta=\theta=0$, one may immediately note, as in Fig. 4(a), that the zero of the numerical dispersion relation does not coincide with that of the model system. The choice of $\bar{q}$ as in (11) corrects this-notice that $\bar{q}$ is indeed an approximation to $q$ in the limit of small grid spacing $h$. The remaining difficulty, as shown in Fig. 4(b), is that in the limit of high wave numbers, the numerical dispersion relation undershoots that of the continuous system - through an appropriate choice of the parameters $\theta$ and $\eta$, the high-frequency characteristics of the scheme may be matched to those of the model system, as shown in Fig. 4(c). Without this extra flexibility of tuning in scheme (10), the results would exhibit a very severe artificial bandlimiting (an extreme form of numerical dispersion). This ability to obtain good accuracy of a scheme over the entire frequency range, using implicit schemes with free parameters has been widely applied in problems in wave mechanics [19].

\section{E. Input/Output and Numerical Connection Conditions}

The two mass-spring systems (7) may also be integrated using similar techniques-here are a pair of schemes

$$
\begin{aligned}
& \delta_{t t} w_{\mathrm{L}}=-\omega_{\mathrm{L}}^{2} \mu_{t, \alpha}\left(w_{\mathrm{L}}-\frac{1}{2} \zeta_{0}\right)-2 \sigma_{\mathrm{L}} \delta_{t} \cdot w_{\mathrm{L}}+F \\
& \delta_{t t} w_{\mathrm{R}}=-\omega_{\mathrm{R}}^{2} \mu_{t, \alpha}\left(w_{\mathrm{R}}-\frac{1}{2} \zeta_{N}\right)-2 \sigma_{\mathrm{R}} \delta_{t} \cdot w_{\mathrm{R}}
\end{aligned}
$$

where $w_{\mathrm{L}}^{n}$ and $w_{\mathrm{L}}^{n}$ are time series. These updates must be connected to scheme (10) through numerical conditions analogous to (8). There are many possible choices, but here are simple conditions which lead to global stability

$$
\begin{aligned}
& u_{0}=u_{1}=0 \quad u_{N}=u_{N-1}=0 \\
& \frac{\theta \kappa k}{\gamma^{2}} \delta_{x-} \zeta_{0}+\mu_{t, \alpha}\left(u_{0}-\delta_{x-} \zeta_{0}-\frac{\omega_{\mathrm{L}}^{2} M_{\mathrm{L}}}{2 \gamma^{2}}\left(w_{\mathrm{L}}-\frac{1}{2} \zeta_{0}\right)\right)=0 \\
& \frac{\theta \kappa k}{\gamma^{2}} \delta_{x+} \zeta_{N}+\mu_{t, \alpha}\left(u_{N}-\delta_{x-} \zeta_{N}+\frac{\omega_{\mathrm{R}}^{2} M_{\mathrm{R}}}{2 \gamma^{2}}\left(w_{\mathrm{R}}-\frac{1}{2} \zeta_{N}\right)\right)=0 .
\end{aligned}
$$




\section{F. Vector-Matrix Form and Computational Complexity}

Scheme (10) is implicit; it is however, linear, and in implementation is best represented as two-step vector matrix update of the form

$$
\mathbf{A} \mathbf{w}^{n+1}=\mathbf{B} \mathbf{w}^{n}+\mathbf{C w}^{n-1}+\mathbf{F}^{n}
$$

where here, the state $\mathbf{w}^{n}$ consists of the grid functions corresponding to the transverse displacement and the longitudinal displacement, and the displacements of the beads, or

$$
\mathbf{w}^{n}=\left[u_{0}^{n}, \ldots, u_{N}^{n}, \zeta_{0}^{n}, \ldots, \zeta_{N}^{n}, w_{\mathrm{L}}^{n}, w_{\mathrm{R}}^{n}\right]^{T}
$$

and $\mathbf{F}^{n}$ is a vector consisting of zeros, except for in the secondlast place, where it scales with the value of the input signal, and is applied to the update of $w_{\mathrm{L}}$.

Though the matrices $\mathbf{A}, \mathbf{B}$ and $\mathbf{C}$ are not given explicitly here, they possess a sparse, nearly block-Toeplitz structure-A is nearly tridiagonal, and $\mathbf{B}$ is nearly pentadiagonal. (By "nearly," one means to within a low rank perturbation, resulting from the choice of numerical boundary conditions.) As the linear system to be solved at each time step will involve the matrix $\mathbf{A}$, various fast methods are available, such as the Thomas algorithm [23], or possibly methods which exploit Toeplitz structure, and which are based on the use of the fast Fourier transform (FFT) and the matrix inversion lemma [24].

Computational complexity for this algorithm scales with $N$, the number of grid points required to represent the state of the spring. The amount of memory required will be approximately $4 \sqrt{q^{2}+f_{\mathrm{s}} \pi / \kappa} / \pi$ units, and the number of operations per time step will scale roughly with the amount of memory, with variations depending on the implementation.

\section{Simulation Results AND COMParison With MEASURED RESPONSES}

Simulation results using scheme (10) are in very good agreement with measured impulse responses taken from real spring reverberation units. See Figs. 5 and 6, which show comparisons between spectrograms of output, as taken from the terminating magnetic bead. The input $F$ to the scheme is an impulse. The scheme is parameterized by physical measurements of the spring materials and geometries, and loss terms are set in a more ad hoc manner, by fitting to observed decay times.

When measuring the impulse response of a particular springreverb tank or one of its constituent springs, a number of factors must be taken into account. Spring reverb unit designs differ in the coils used for input and output into the spring system, and therefore it is necessary to either provide input and output loads of an appropriate impedance or use an intermediate transformer to provide impedance-coupling. The units measured for this publication were of designs such that a standard power-amplifier was appropriate for driving of the system, whilst a lownoise microphone preamplifier was sufficient for recording the signal produced at the output coil. Naive methods of impulse response measurement are unsuitable for use in this context, as
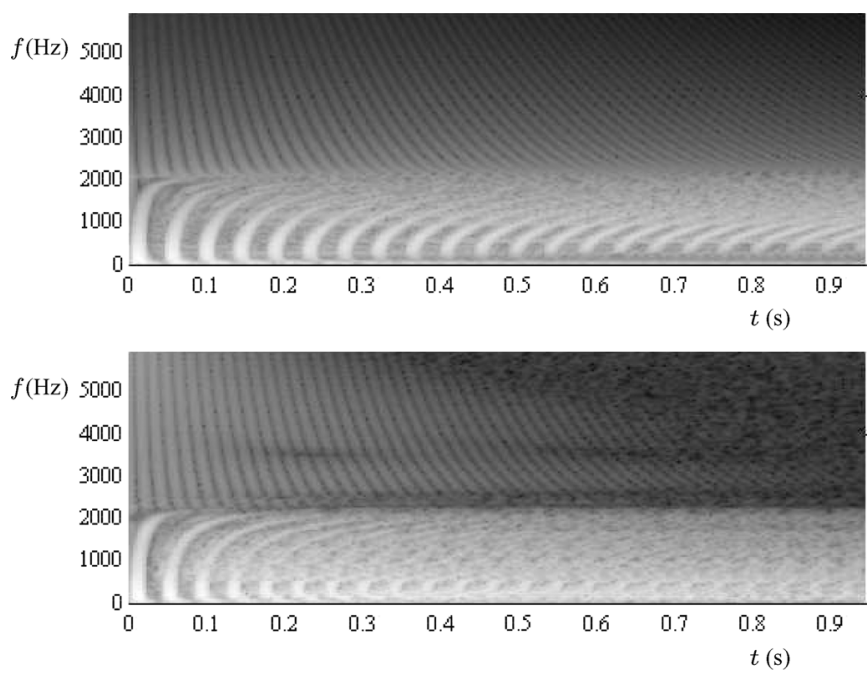

Fig. 5. Spectrogram of measured output from a single spring (bottom) and simulated results (top). Here, the spring has parameters $\kappa=0.068, q=835$, and $\gamma=1980$, and scheme (10) is used, at $44.1 \mathrm{kHz}$, with the parameter settings $\eta=0.411$ and $\theta=0.000411$.
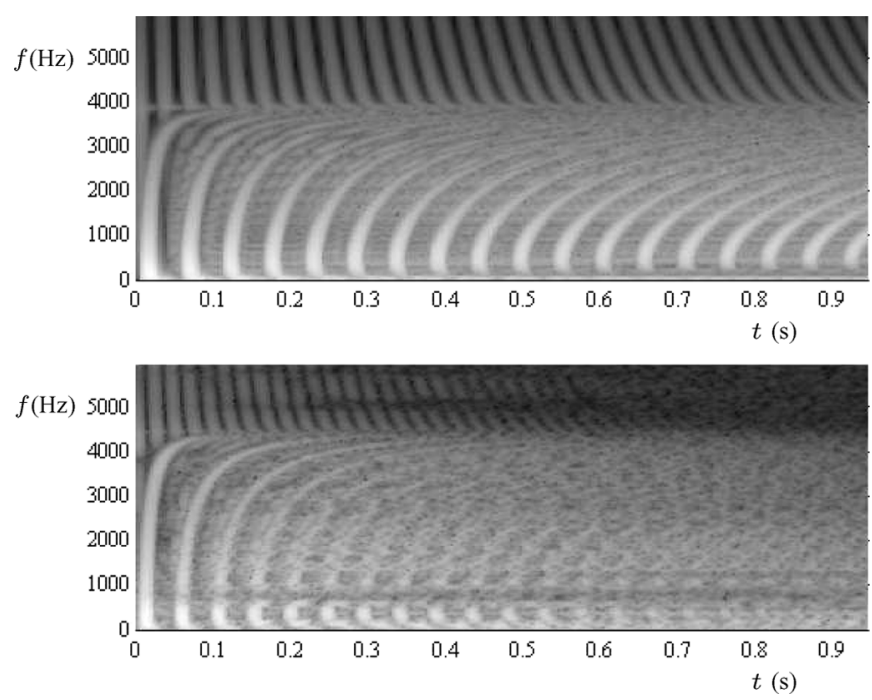

Fig. 6. Spectrogram of measured output from a single spring (bottom) and simulated results (top). Here, the spring has parameters $\kappa=0.0246, q=1905$, and $\gamma=1190$, and scheme (10) is used, at $44.1 \mathrm{kHz}$, with the parameter settings $\eta=0.09$ and $\theta=0.00022$.

producing an impulsive input signal with enough energy to excite the system to a satisfactory level above the noise floor is difficult. Instead, an impulse response measurement method using a sine-sweep was used [25]. To obtain the response of a single spring in a unit containing several springs, padded clamps were used to suppress vibration in the springs not under consideration.

In Fig. 5, a comparison of measured and simulated responses is shown, which for a spring of a relatively low transition frequency $f_{c}$. In this case, the device is an Olson X-82 Reverberation Unit, which is a small OEM reverb tank intended for use in guitar amplifiers; this unit is mostly equivalent to the well-known Accutronics Type 1 unit. The rates of reflections of individual pulses, both above and below the transition frequency are correct, as is the dispersive behavior of propagation, 
easily seen as "bends" in the arcs in the plots, which represent individual reflections. Notice that the first few reflections are simulated with very good accuracy. The behavior of the scheme remains very good above the transition frequency $f_{\mathrm{c}}$, but there is clearly some additional complexity in the real response below $f_{\mathrm{c}}$, especially after several repeated reflections, that is not captured by the model; this is most likely related to the unmodeled other transverse polarization, and could also result from the choice of clamped boundary conditions in the transverse polarization. It is also clear that the loss model, which is grossly correct, does not capture some variations in the damping rates, particularly above the transition frequency.

When the transition frequency $f_{\mathrm{c}}$ becomes high (on the order of approximately $4000 \mathrm{~Hz}$ ), it becomes more difficult to tune the scheme such that the dispersion relation is matched over the whole spectrum; such is the case for another unit, taken from a Leem Pro KA-1210 guitar amplifier, which is mostly equivalent to the Accutronics Type 8 unit. In particular, notice from Fig. 6 that the transition frequency $f_{\mathrm{c}}$ is slightly undershot by the scheme, and that the reflections are more widely separated in the region above $f_{\mathrm{c}}$.

Computation time is long in Matlab, but not extreme and scales roughly with $f_{s} / \kappa$. For the spring of parameters as given in the caption to Fig. 5, it takes approximately $40 \mathrm{~s}$ to generate $1 \mathrm{~s}$ of output, on a Dell D810 laptop with a 2-GHz Pentium processor. For the spring of parameters as given in the caption to Fig. 6, it requires approximately $160 \mathrm{~s}$ to generate $1 \mathrm{~s}$ of output. A port to a $\mathrm{C}$ environment will allow for much faster computation times.

Sound examples corresponding to the two spring configurations presented here are available on the author's website at http://www.ccrma.stanford.edu/ bilbao/soundex/spring/

\section{CONCLUSION}

The finite difference simulation scheme presented in this article offers a great deal of flexibility - the model parameters are very few in number (five for the spring itself, and a handful more for the mass-spring terminations), allowing intuitive virtual reverb design. This is, of course, a benefit of any physical model. Computational expense is higher, but limited by the model parameters and the choice of sample rate, so one may expect that computational cost will become less and less of a concern in future years. Another difficulty lies at the level of algorithm design - standard explicit finite difference schemes are not a good match to this system, and some involved design work is necessary in order to obtain an acceptable simulated result.

Many improvements are possible at the level of the spring model itself. System (2), while tractable, neglects various effects which will impact on the dispersion profile. One perhaps important such effect is that of the unmodeled other transverse polarization; while, for a bar of symmetric cross-section, and without curvature, the two polarizations are degenerate, when there is curvature, this degeneracy is broken, leading to a splitting of the dispersion curve. As there will then be two closely-spaced dispersion curves, one can expect audible beating effects to occur. The other major issue is the modeling of loss, which has been carried out here in an ad hoc manner, and which could be considerably improved, along the lines of models used in problems in plate vibration in musical acoustics [26].

Other improvements rely on the availability of good measured data - this is particularly true in the case of the loss model for the spring, as well as in the case of the terminating beads. While it is easy enough to determine the mass of the beads, it is more difficult to determine the characteristic frequencies $\omega_{\mathrm{L}}$ and $\omega_{\mathrm{R}}$, and the damping parameters $\sigma_{\mathrm{L}}$ and $\sigma_{\mathrm{L}}$. In some spring reverb configurations, the beads operate in a highly over-damped mode, leading to a very wide resonance, and thus susceptibility to the entire spectrum of input frequencies.

The model presented here could be extended to the case of multiple springs - the usual case in "tank"-type reverberation units [12]. A model of a parallel driven set of springs follows immediately from the discussion here, but the case of the series connection is more delicate- the nature of the connection must be specified, and, in simulation, two difference schemes, of distinct parameters must be coupled at the connection point.

\section{APPENDIX \\ ENERGY-BASED STABILITY ANALYSIS}

System (2), terminated by boundary conditions as in (8), and coupled with the equations of motion (7) of the terminating mass-spring systems, possesses an energy dissipation property which is very useful in coming to conclusions about numerical stability of associated schemes, as well as in arriving at provably stable numerical boundary conditions. In this short appendix, only the problem of the scheme for the spring of infinite extent will be considered - the problem of the finite case, including the connection to mass-spring elements, and of the determination of numerically stable connection conditions will not be covered here; though not extremely different from the infinite case, the algebraic machinery becomes quite involved, and the main ideas are somewhat obscured-see [15] for a full treatment of the relevant ideas. A short sketch appears at the end of this appendix.

\section{A. Continuous Case}

An $L_{2}$ spatial inner product and norm, over the real line $x \in \mathbb{R}$ may be defined, for two functions $f(x)$ and $g(x)$, as

$$
\langle f, g\rangle=\int_{-\infty}^{\infty} f g d x \quad\|f\|=\sqrt{\langle f, f\rangle} .
$$

Integration by parts may be written as

$$
\left\langle f, g_{x}\right\rangle=-\left\langle f_{x}, g\right\rangle
$$

Taking the inner product of (2a) and (2b) with $u_{t}$ and $q^{2} \zeta_{t}$ respectively leads, after repeated applications of integration by parts, from (30), to the following energy balance:

$$
\dot{\mathfrak{H}}=-\mathfrak{Q}
$$


with

$$
\mathfrak{H}=\mathfrak{T}+\mathfrak{V}_{\mathrm{uc}}+\mathfrak{V}_{\times}
$$

and

$$
\begin{aligned}
\mathfrak{T} & =\frac{1}{2}\left\|u_{t}\right\|^{2}+\frac{q^{2}}{2}\left\|\zeta_{t}\right\|^{2} \\
\mathfrak{V}_{\mathrm{uc}} & =\frac{\kappa^{2}}{2}\left\|u_{x x}+q^{2} u\right\|^{2} \quad \mathfrak{V}_{\times}=\frac{\gamma^{2} q^{2}}{2}\left\|\zeta_{x}-u\right\|^{2} \\
\mathfrak{Q} & =2 \sigma_{\mathrm{t}}\left\|u_{t}\right\|^{2}+2 q^{2} \sigma_{1}\left\|\zeta_{t}\right\|^{2} .
\end{aligned}
$$

Here, $\mathfrak{H}$ scales with the total energy of the spring, $\mathfrak{T}$ with the kinetic energy, and $\mathfrak{V}_{\text {uc }}$ and $\mathfrak{V}_{\times}$with contributions to the potential energy due to uncoupled and coupled motion, respectively. $\mathfrak{Q}$ represents loss. Notice in particular that $\mathfrak{H}, \mathfrak{Q} \geq 0$.

The system is thus strictly dissipative, so that

$$
0 \leq \mathfrak{H}\left(t_{2}\right) \leq \mathfrak{H}\left(t_{1}\right) \quad \text { when } \quad t_{2} \geq t_{1} .
$$

The energy thus decreases montonically; as such, it may be used as a Lyapunov function in order to bound the state of the solution-see [15] for more on this topic.

\section{B. Discrete Energy Identities and Inequalities}

The idea behind energy-based techniques as applied to numerical methods is to find a similar dissipative property for a scheme. One must first find a discrete energy balance analogous to (18), and then determine conditions under which the numerical energy is non-negative, which become stability conditions for the scheme. One may proceed in steps similar to those above for the continuous system, by first defining an inner product and norm over a discrete space. For two grid functions $f_{l}$ and $g_{l}$, for $l \in \mathbb{Z}$, where $\mathbb{Z}$ is the set of integers, and where $h$ is the grid spacing

$$
\langle f, g\rangle=\sum_{l \in \mathbb{Z}} h f_{l} g_{l} \quad\|f\|=\sqrt{\langle f, f\rangle} .
$$

Various identities involving time difference operators are of use. Defining the forward and backward operators, in terms of the unit shifts from (9), as

$$
\delta_{t+}=\frac{1}{k}\left(e_{t+}-1\right) \quad \delta_{t-}=\frac{1}{k}\left(1-e_{t-}\right)
$$

it is true, for any grid function $f_{l}^{n}$, that

$$
\begin{aligned}
\left\langle\delta_{t} . f, \delta_{t t} f\right\rangle & =\delta_{t+}\left(\frac{1}{2}\left\|\delta_{t-} f\right\|^{2}\right) \\
\left\langle\delta_{t} . f, f\right\rangle & =\delta_{t+}\left(\frac{1}{2}\left\langle f, e_{t-} f\right\rangle\right)
\end{aligned}
$$

and that

$$
\left\langle\delta_{t} . f, \mu_{t, \alpha} f\right\rangle=\delta_{t+}\left(\frac{\alpha}{2}\left\langle f, e_{t-} f\right\rangle+\frac{1-\alpha}{2} \mu_{t-}\|f\|^{2}\right) .
$$

Furthermore, the quantity in brackets above is bounded as

$$
\frac{\alpha}{2}\left\langle f, e_{t-} f\right\rangle+\frac{1-\alpha}{2} \mu_{t-}\|f\|^{2} \geq 0 \quad \text { if } \quad 0 \leq \alpha \leq \frac{1}{2} .
$$

It is also true that

$$
\left\langle f, e_{t-} f\right\rangle \geq-\frac{k^{2}}{4}\left\|\delta_{t-} f\right\|^{2} .
$$

Summation by parts may be written as

$$
\left\langle f, \delta_{x+g} g\right\rangle=-\left\langle\delta_{x-} f, g\right\rangle .
$$

There are numerous bounds which are of use in the setting of energy analysis. For example, it is true, for any grid function $f_{l}$, that

$$
\left\|\delta_{x+f}\right\| \leq \frac{2}{h}\|f\| .
$$

For the special problem of the spring, the following bound is also useful:

$$
\left\|\left(\delta_{x x}+q^{2}\right) f\right\| \leq \frac{2+\left|q^{2} h^{2}-2\right|}{h^{2}}\|f\|
$$

for any real $q$.

\section{Discrete Case}

Taking the inner products of (10a) and (10b) with $\delta_{t} . u$ and $q^{2} \delta_{t} . \zeta$, adding the resulting equations, and making use of identities (24), (20), and (21) leads to

$$
\delta_{t+} \mathfrak{h}=-\mathfrak{q}
$$

with

$$
\mathfrak{h}=\mathfrak{t}+\mathfrak{v}_{\mathrm{uc}}+\mathfrak{v}_{\times}
$$

and

$$
\begin{aligned}
\mathfrak{t}= & \frac{1}{2}\left\|\delta_{t-} u\right\|^{2}+\frac{q^{2}}{2}\left\|\delta_{t-} \zeta\right\|^{2}-\frac{\eta \kappa k}{2}\left\|\delta_{x+} \delta_{t-} u\right\|^{2} \\
& -\frac{\theta q^{2} \gamma^{2} k^{2}}{2}\left\|\delta_{x+} \delta_{t-} \zeta\right\|^{2} \\
\mathfrak{v}_{\mathrm{uc}}= & \frac{\kappa^{2}}{2}\left\langle\left(\delta_{x x}+\bar{q}^{2}\right) u, e_{t-}\left(\delta_{x x}+\bar{q}^{2}\right) u\right\rangle \\
\mathfrak{v}_{\times}= & \frac{\gamma^{2} q^{2}}{2}\left[(1-\alpha)\left\|\delta_{x+} \zeta-u\right\|^{2}\right. \\
& \left.+\alpha\left\langle\delta_{x+} \zeta-u, e_{t-}\left(\delta_{x+} \zeta-u\right)\right\rangle\right] \\
\mathfrak{q}= & 2 \sigma_{\mathrm{t}}\left\|\delta_{t} \cdot u\right\|^{2}+2 q^{2} \sigma_{1}\left\|\delta_{t} \cdot \zeta\right\|^{2} .
\end{aligned}
$$

Thus, an energy balance, analogous to (18) exists for scheme (10). Note however, that the energy $\mathfrak{h}$ is made up of components of indeterminate sign; the loss term $\mathfrak{q}$, however, is non-negative, so it remains to determine conditions under which the energy 
itself is non-negative, in which case it will be monotonically decreasing.

First, note that when $0 \leq \alpha \leq 1 / 2$, the contribution $\mathfrak{v}_{\times}$is non-negative, from inequality (22). Under these conditions, then

$$
\mathfrak{h} \geq \mathfrak{t}+\mathfrak{v}_{\mathrm{uc}}
$$

But $\mathfrak{t}$ may be bounded from below, using (25) as

$\mathfrak{t} \geq \frac{1}{2}\left(1-\frac{4 \eta^{+} \kappa k}{h^{2}}\right)\left\|\delta_{t-u}\right\|^{2}+\frac{q^{2}}{2}\left(1-\frac{4 \theta^{+} \gamma^{2} k^{2}}{h^{2}}\right) \| \delta_{t-\zeta \|^{2}}$

Using (23) and (26), $\mathfrak{v}_{\mathrm{uc}}$ may also be bounded from below as

$$
\begin{aligned}
\mathfrak{v}_{\mathrm{uc}} & \geq-\frac{\kappa^{2} k^{2}}{8}\left\|\left(\delta_{x x}+\bar{q}^{2}\right) \delta_{t-} u\right\|^{2} \\
& \geq-\frac{\kappa^{2} k^{2}\left(1+\left|1-\bar{q}^{2} h^{2} / 2\right|\right)^{2}}{2 h^{4}}\left\|\delta_{t-u} u\right\|^{2}
\end{aligned}
$$

or, using the special choice of $\bar{q}$ as in (11), as

$$
\mathfrak{v}_{\mathrm{uc}} \geq-\frac{\kappa^{2} k^{2}(1+|\cos (q h)|)^{2}}{2 h^{4}}\left\|\delta_{t-} u\right\|^{2}
$$

Finally, the energy $\mathfrak{h}$ may be bounded from below as

$$
\begin{aligned}
\mathfrak{h} \geq \frac{q^{2}}{2} & \left(1-\frac{4 \theta^{+} \gamma^{2} k^{2}}{h^{2}}\right) \| \delta_{t-\zeta \|^{2}} \\
& +\frac{1}{2}\left(1-\frac{4 \eta^{+} \kappa k}{h^{2}}-\frac{\kappa^{2} k^{2}(1+|\cos (q h)|)^{2}}{h^{4}}\right)\left\|\delta_{t-u}\right\|^{2}
\end{aligned}
$$

which is non-negative under conditions (12). Under these conditions, given that the loss term $\mathfrak{q}$ is non-negative, it is true that

$$
0 \leq \mathfrak{h}^{n_{1}} \leq \mathfrak{h}^{n_{2}} \quad \text { for } \quad n_{2} \geq n_{1} \geq 0
$$

and the scheme is thus dissipative in a discrete sense.

\section{Boundary Conditions}

In this short section, connection conditions to the terminating mass-spring systems are derived using energy methods - stable numerical connection conditions may be obtained through the application of analogous techniques to scheme (10).

First, the definitions of the inner product and norm, restricted to the unit interval, may be altered from (16) as

$$
\langle f, g\rangle=\int_{0}^{1} f g d x \quad\|f\|=\sqrt{\langle f, f\rangle}
$$

Integration by parts may be written as

$$
\left\langle f, g_{x}\right\rangle=-\left\langle f_{x}, g\right\rangle+\left.f g\right|_{0} ^{1}
$$

The energy balance from (18) may be generalized as

$$
\dot{\mathfrak{H}}=-\mathfrak{Q}+\mathfrak{B}
$$

where $\mathfrak{H}$ and $\mathfrak{Q}$ are as defined for the continuous system, now over the unit interval. The boundary term $\mathfrak{B}$ is given by

$$
\begin{aligned}
\mathfrak{B}=\left(\gamma^{2} q^{2}\left(\zeta_{x}-u\right)-\kappa^{2} u_{t}\left(u_{x x x}+\right.\right. & \left.q^{2} u_{x}\right) \\
& \left.+\kappa^{2} u_{t x}\left(u_{x x}+q^{2} u\right)\right)\left.\right|_{0} ^{1}
\end{aligned}
$$

Under the first two of boundary conditions (8), which apply to the transverse displacement $u$ alone, this expression simplifies to

$$
\mathfrak{B}=\left.\gamma^{2} q^{2}\left(\zeta_{x}-u\right)\right|_{0} ^{1}
$$

One may derive energy balances for the mass-spring systems themselves, by multiplying (7a) and (7b) by $M_{\mathrm{L}} q^{2} \dot{w}_{\mathrm{L}}$ and $M_{\mathrm{R}} q^{2} \dot{w}_{\mathrm{R}}$ respectively, to get

$$
\dot{\mathfrak{H}}_{\mathrm{L}}=\mathfrak{B}_{\mathrm{L}}-\mathfrak{Q}_{\mathrm{L}}+\mathfrak{P}_{\text {in }} \quad \dot{\mathfrak{H}}_{\mathrm{R}}=\mathfrak{B}_{\mathrm{R}}-\mathfrak{Q}_{\mathrm{R}}
$$

with

$$
\mathfrak{H}_{\mathrm{L}}=\mathfrak{T}_{\mathrm{L}}+\mathfrak{V}_{\mathrm{L}} \quad \mathfrak{H}_{\mathrm{R}}=\mathfrak{T}_{\mathrm{R}}+\mathfrak{V}_{\mathrm{R}}
$$

where

$$
\begin{aligned}
& \mathfrak{T}_{\mathrm{L}}=\frac{M_{\mathrm{L}} q^{2} \dot{w}_{\mathrm{L}}^{2}}{2} \quad \mathfrak{V}_{\mathrm{L}}=\frac{M_{\mathrm{L}} \omega_{\mathrm{L}}^{2} q^{2}}{4}\left(w_{\mathrm{L}}^{2}+\left(w_{\mathrm{L}}-\zeta(0, t)\right)^{2}\right) \\
& \mathfrak{T}_{\mathrm{R}}=\frac{M_{\mathrm{R}} q^{2} \dot{w}_{\mathrm{R}}^{2}}{2} \quad \mathfrak{V}_{\mathrm{R}}=\frac{M_{\mathrm{R}} \omega_{\mathrm{R}}^{2} q^{2}}{4}\left(w_{\mathrm{R}}^{2}+\left(w_{\mathrm{R}}-\zeta(1, t)\right)^{2}\right) \\
& \mathfrak{Q}_{\mathrm{L}}=2 M_{\mathrm{L}} q^{2} \sigma_{\mathrm{L}} \dot{w}_{\mathrm{L}}^{2} \quad \mathfrak{Q}_{\mathrm{R}}=2 M_{\mathrm{R}} q^{2} \sigma_{\mathrm{R}} \dot{w}_{\mathrm{R}}^{2} \\
& \mathfrak{B}_{\mathrm{L}}=-\frac{M_{\mathrm{L}} q^{2} \omega_{\mathrm{L}}^{2}}{2} \zeta_{t}(0, t)\left(w_{\mathrm{L}}-\zeta(0, t)\right) \\
& \mathfrak{B}_{\mathrm{R}}=-\frac{M_{\mathrm{R}} q^{2} \omega_{\mathrm{R}}^{2}}{2} \zeta_{t}(1, t)\left(w_{\mathrm{L}}-\zeta(1, t)\right) \\
& \mathfrak{P}_{\mathrm{in}}=M_{\mathrm{L}} q^{2} \dot{w}_{\mathrm{L}} F .
\end{aligned}
$$

Again, $\mathfrak{T}_{\mathrm{L}}\left(\mathfrak{T}_{\mathrm{R}}\right), \mathfrak{V}_{\mathrm{L}}\left(\mathfrak{V}_{\mathrm{R}}\right)$, and $\mathfrak{Q}_{\mathrm{L}}\left(\mathfrak{Q}_{\mathrm{R}}\right)$ represent the kinetic energy, potential energy and loss for the left (right) mass-spring system. $\mathfrak{B}_{\mathrm{L}}$ and $\mathfrak{B}_{\mathrm{R}}$ scale with the power supplied to the massspring systems, and $\mathfrak{P}_{\text {in }}$ is the power supplied by the excitation. 
Combining the energy balances (18) and (33) for the spring and the terminations leads to the global energy balance

$$
\dot{\mathfrak{H}}_{\text {total }}=\mathfrak{B}_{\text {total }}-\mathfrak{Q}_{\text {total }}+\mathfrak{P}_{\text {in }}
$$

where $\mathfrak{H}_{\text {total }}=\mathfrak{H}+\mathfrak{H}_{\mathrm{L}}+\mathfrak{H}_{\mathrm{R}}, \mathfrak{Q}_{\text {total }}=\mathfrak{Q}+\mathfrak{Q}_{\mathrm{L}}+\mathfrak{Q}_{\mathrm{R}}$ and $\mathfrak{B}_{\text {total }}=\mathfrak{B}+\mathfrak{B}_{\mathrm{L}}+\mathfrak{B}_{\mathrm{R}}$. Under the boundary conditions (8), the total boundary term $\mathfrak{B}_{\text {total }}$ vanishes, and, due to the non-negativity of $\mathfrak{H}_{\text {total }}$ and $\mathfrak{Q}_{\text {total }}$, it is then true that $\dot{\mathfrak{H}} \leq \mathfrak{P}_{\text {in }}$. In other words, the total energy of the system may be bounded in terms of the time integral of the supplied power. Clearly, under transient conditions, (i.e., when $\mathfrak{P}_{\text {in }}=0$ ), a dissipativity property follows, and it is possible to bound the state of the system in terms of the initial conditions.

\section{REFERENCES}

[1] T. Stilson and J. O. Smith, III, "Analyzing the Moog VCF with considerations for digital implementation," in Proc. Int. Comput. Music Conf., Hong Kong, Aug. 1996, pp. 398-401.

[2] T. Hélie, "On the use of Volterra series for real-time simulations of weakly nonlinear analog audio devices: Application to the Moog ladder filter," in Proc. 9th Int. Digital Audio Effects Conf., Montreal, QC, Canada, Sep. 2006, pp. 7-12.

[3] V. Välimäki and A. Huovilainen, "Oscillator and filter algorithms for virtual analog synthesis," Comput. Music J., vol. 30, no. 2, pp. 19-31, 2006.

[4] D. Yeh, J. Abel, A. Vladimirescu, and J. O. Smith, III, "Numerical methods for simulation of guitar distortion circuits," Comput. Music J., vol. 32, no. 2, pp. 23-42, 2008.

[5] S. Bilbao, K. Arcas, and A. Chaigne, "A physical model of plate reverberation," in Proc. IEEE Int. Conf. Acoust., Speech, Signal Process., Toulouse, France, 2006, vol. 5, pp. 165-168.

[6] S. Bilbao, "A digital plate reverberation algorithm," J. Audio Eng. Soc., vol. 55, no. 3, pp. 135-144, 2007.

[7] K. Arcas, "Simulation numérique d'un réverbérateur à plaque," Ph.D. dissertation, Ecole Nationale Supérieure de Techniques Avancées, Palaiseau, France, 2008.

[8] J. O. Smith, III, S. Serafin, and D. Berners, "Doppler simulation and the Leslie," in Proc. 5th Int. Conf. Digital Audio Effects, Hamburg, Germany, 2002, pp. 13-20.

[9] J. Abel, D. Berners, S. Costello, and J. O. Smith, III, "Spring reverb emulation using dispersive allpass filters in a waveguide structure," presented at the 121st Audio Engineering Society Convention, San Francisco, CA, Oct. 2006, Preprint 6954.

[10] J. Parker, "Spring reverberation: A finite difference approach," M.S. thesis, Univ. of Edinburgh, Edinburgh, U.K., 2008.

[11] L. Hammond, "Electical Musical Instrument," U.S. Patent 2,230,836, Feb. 2, 1941
[12] A. C. Young and P. It, "Artificial Reverberation Unit," U.S. Patent 3,106,610, Oct. 8, 1963.

[13] J. D. Stack, "Sound Reverberating Device," U.S. Patent 2,437,445, Mar. 9, 1948.

[14] R. Kuroki, "Sound Effect Imparting Apparatus," U.S. Patent 6,580,796, Jan. 26, 1999.

[15] S. Bilbao, Numerical Sound Synthesis. Chichester, U.K.: Wiley, 2009 , to be published.

[16] W. Wittrick, "On elastic wave propagation in helical springs," Int. J. Mech. Sci., vol. 8, pp. 25-47, 1966.

[17] N. Fletcher, T. Tarnopolskaya, and F. de Hoog, "Wave propagation on helices and hyperhelices: A fractal regression," Proc. R. Soc., vol. 457, pp. 33-43, 2001.

[18] K. Graff, Wave Motion in Elastic Solids. New York: Dover, 1975.

[19] S. Lele, "Compact finite difference schemes with spectral-like resolution," J. Comput. Phys., vol. 103, pp. 16-42, 1992.

[20] J. Strikwerda, Finite Difference Schemes and Partial Differential Equations. Pacific Grove, CA: Wadsworth and Brooks/Cole, 1989.

[21] B. Gustaffson, H.-O. Kreiss, and J. Oliger, Time Dependent Problems and Difference Methods. New York: Wiley, 1995.

[22] A. Tveito and R. Winther, Introduction to Partial Differential Equations. New York: Springer, 1998.

[23] L. H. Thomas, "Elliptic problems in linear difference equations over a network," Watson Sci. Comput. Lab., Columbia Univ., New York, 1949, Tech. Rep..

[24] R. Horn and C. Johnson, Matrix Analysis. Cambridge, U.K.: Cambridge Univ. Press, 1985.

[25] A. Farina, "Simultaneous measurement of impulse response and distortion with a swept-sine technique," presented at the 108th Audio Eng. Soc. Conv., Paris, France, Feb. 2000, Preprint 5093.

[26] C. Lambourg, A. Chaigne, and D. Matignon, "Time-domain simulation of damped impacted plates. II Numerical model and results," J. Acoust. Soc. Amer., vol. 109, no. 4, pp. 1433-1447, 2001.

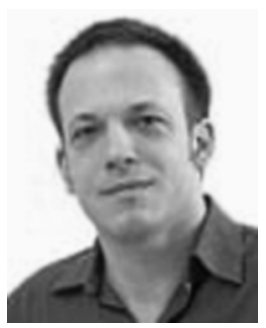

Stefan Bilbao received the B.A. degree in physics from Harvard University, Cambridge, MA, in 1992 and the M.Sc. and Ph.D. degrees in electrical engineering from Stanford University, Stanford, CA, in 1996 and 2001, respectively.

$\mathrm{He}$ is currently a Senior Lecturer in the Acoustics and Fluid Dynamics Group/Music subject area, University of Edinburgh, Edinburgh, U.K., and was previously a Lecturer at the Sonic Arts Research Centre, Queen's University Belfast, and a Research Associate at the Stanford Space Telecommunications and Radioscience Laboratories.

Julian Parker received the B.A. degree in natural sciences from Cambridge University, Cambridge, U.K., in 2005 and the M.Sc. degree in acoustics and music technology, University of Edinburgh, Edinburgh, U.K., in 2008.

$\mathrm{He}$ is currently working as a Researcher within the Acoustics and Audio Signal Processing Group at Helsinki University of Technology, Helsinki, Finland. 